\title{
Spotlight on aerobic glycolysis
}

In the brain, glucose acts through various metabolic pathways to produce energy - its most important function in the brain - but also acts through several other crucial regulatory (for example, apoptotic), protective (that is, against reactive oxygen species) and anabolic (for example, protein- and lipidsynthesizing) pathways. The most efficient pathway for energy generation involves oxidative phosphorylation, but glucose can also quickly and efficiently deliver energy directly to membrane processes, such as ion pumps. When glucose acts independently of oxidative phosphorylation in performing these functions it is traditionally referred to as aerobic glycolysis. Aerobic glycolysis has been relatively under-researched in the brain but, in two studies, Raichle and colleagues now show that there is regional variation in this process in the resting brain and that high aerobic glycolysis might be associated with deposition of amyloid- $\beta$ (that is, plaques) later in life.

In the first study, the authors used positron emission tomography (PET) to measure the metabolic rates for oxygen (in three scans using $\left[{ }^{15} \mathrm{O}\right]$-labelled water, carbon monoxide or oxygen) and glucose (using $\left[{ }^{18} \mathrm{~F}\right]$-labelled fluorodeoxglucose) in young adults. They found high rates of aerobic glycolysis in the prefrontal cortex, lateral parietal cortex, precuneus and posterior cingulate cortex, lateral temporal cortex, gyrus rectus, and caudate nuclei. These regions correspond with areas comprising the 'default mode network' - a set of regions that are most active when an individual is awake but not engaged in a task — and cognitive control networks.
The cerebellum and the inferior temporal gyrus, including the hippocampus, showed low rates of aerobic glycolysis.

Amyloid- $\beta$ plaques are found in regions of the default mode network early in Alzheimer's disease. In the second study, Raichle and colleagues therefore investigated whether the reliance on aerobic glycolysis in these areas is related to amyloid- $\beta$ deposition. They used PET to assess amyloid- $\beta$ deposition in patients with Alzheimer's disease and in cognitively normal people who were known to have elevated levels of the protein, and compared the resulting brain images with the spatial distribution maps of aerobic glycolysis levels from the first study. The authors divided the brain images into 167 nonoverlapping cubes and, in a second approach, picked 60 small spherical areas in several regions of interest. For each cube and sphere, they assessed the levels of amyloid- $\beta$ and aerobic glycolysis. Both methods revealed a striking spatial correlation between levels of amyloid- $\beta$ and aerobic glycolysis, and this correlation was stronger in people with Alzheimer's disease than in cognitive normal people with elevated amyloid- $\beta$ levels.

It is not currently known what underlies the observed regional variations in aerobic glycolysis, but the authors suggest that they may be related to regional differences in energy requirements for synaptic processes, anabolic reactions and/or regulation of redox states. As some glucose-dependent synaptic processes as well as oxidative stress are known to be impaired in Alzheimer's disease, these findings indicate that a reliance on aerobic glycolysis might render a brain area vulnerable to pathogenic processes leading to Alzheimer's disease. They also suggest that aerobic glycolysis has potential as a biomarker for the early detection of the disease.

Leonie Welberg

ORIGINAL RESEARCH PAPERS Vaishnavi, S. N. et al. Regional aerobic glycolysis in the human brain. Proc. Natl Acad. Sci. USA 13 Sep 2010 (doi: 10.1073/pnas.1010459107)| Vlassenko, A. G. et al. Spatial correlation between brain aerobic glycolysis and amyloid- $\beta$ deposition. Proc. Natl Acad. Sci. USA 13 Sep 2010 (doi: 10.1073/pnas.1010461107)

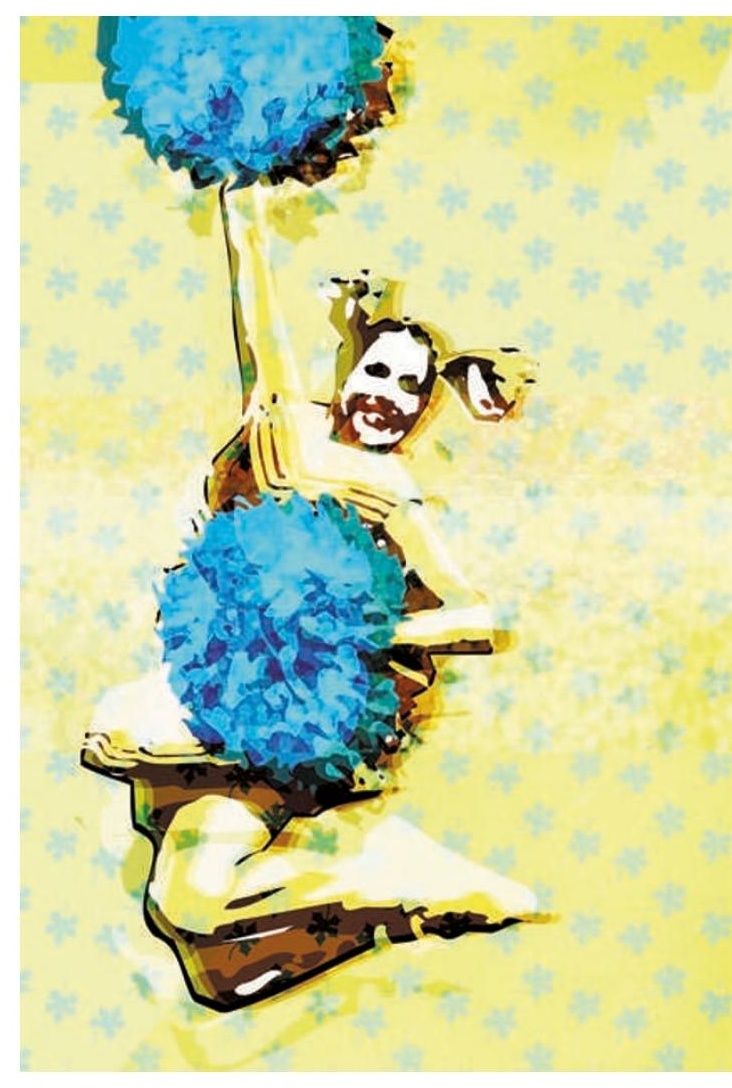

